\title{
Mitochondrial Cardiomyopathy with a Unique ${ }^{99 \mathrm{~m}}$ Tc-MIBI/ ${ }^{123}$ I-BMIPP Mismatch Pattern
}

\author{
Ryosuke Tashiro $^{1}$, Noriko Onoue ${ }^{1}$, Hiroya Rikimaru ${ }^{2}$, Kenichi Tsukita ${ }^{3}$, Hiroshi Fujita ${ }^{1}$, \\ Nobuhiro Yamaguchi ${ }^{1}$, Takeshi Ishizuka ${ }^{1}$, Yasushi Suzuki ${ }^{3}$, \\ Hiroyoshi Suzuki ${ }^{4}$ and Tsuyoshi Shinozaki ${ }^{1}$
}

\begin{abstract}
A 42-year-old man was referred to our hospital due to chest pain, diabetes mellitus, and sensorineural hearing loss. Transthoracic echocardiography revealed diffuse left ventricular hypokinesis. He was diagnosed with mitochondrial disease and a c.A3243G mutation was identified in his mitochondrial DNA. This case of mitochondrial cardiomyopathy demonstrated a low uptake of ${ }^{123} \mathrm{I}$-BMIPP, while the uptake of ${ }^{99 \mathrm{~m}} \mathrm{Tc}$-MIBI was preserved. In contrast, previous reports have noted the increased uptake of ${ }^{123} \mathrm{I}$-BMIPP and the decreased uptake of ${ }^{99 \mathrm{~m}} \mathrm{Tc}-\mathrm{MIBI}$. This is the first study to show this unique ${ }^{99 \mathrm{~m}} \mathrm{Tc}-\mathrm{MIBI} /{ }^{123} \mathrm{I}$-BMIPP mismatch pattern. We also discuss the relationships among the cardiac scintigraphy, cardiac magnetic resonance imaging, and histopathology findings.
\end{abstract}

Key words: mitochondrial cardiomyopathy, ${ }^{99 \mathrm{~m}} \mathrm{Tc}-\mathrm{MIBI},{ }^{123} \mathrm{I}-\mathrm{BMIPP}$, cardiac magnetic resonance, late gadolinium enhancement

(Intern Med 56: 321-325, 2017)

(DOI: 10.2169/internalmedicine.56.7525)

\section{Introduction}

Mitochondrial cardiomyopathy can manifest with various cardiac phenotypes, such as left ventricle (LV) hypertrophy, dilatation, wall motion abnormality, and lethal ventricular arrhythmia; these symptoms range from subclinical to critical conditions (1). The severity of myocardial dysfunction also predicts the prognosis of patients with mitochondrial disease, which highlights the importance of noninvasive assessment for myocardial dysfunction. Myocardial perfusion/metabolism mismatch (the decreased uptake of technetium-99m methoxyisobutylisonitrile $\left[{ }^{99 \mathrm{~m}} \mathrm{Tc}-\mathrm{MIBI}\right] /$ the increased uptake of 123-labeled 15-4-iodophenyl-3-(R, S)-methyl-pentadecanoic acid [ $\left.\left.{ }^{123} \mathrm{I}-\mathrm{BMIPP}\right]\right)$ has been reported in patients with mitochondrial cardiomyopathy $(2,3)$. However, the previous studies did not compare the morphological images of patients with this condition.
In the present case, we compared images of myocardial perfusion $\left({ }^{99 m} \mathrm{Tc}-\mathrm{MIBI}\right.$ scintigraphy), fatty acid metabolism ( ${ }^{123}$ I-BMIPP scintigraphy), and morphology (cardiac magnetic resonance [CMR] and histology), and found that the images of our patient, who had a mismatch between perfusion and metabolism, were unique from those in the previous reports.

\section{Case Report}

A 42-year-old man $(157 \mathrm{~cm}, 48.4 \mathrm{~kg})$ was referred to our hospital with left chest pain, diabetes mellitus, and sensorineural hearing loss. His mother had diabetes mellitus; however, she did not have hearing loss or cardiovascular disease. At admission, his blood pressure was $121 / 79 \mathrm{mmHg}$, his heart rate was 82 beats/min with a regular pulse, and his percutaneous oxygen saturation was $98 \%$ in room air. Although systolic heart murmurs were audible at the base

\footnotetext{
${ }^{1}$ Division of Cardiology, Sendai Medical Center, National Hospital Organization, Japan, ${ }^{2}$ Division of Radiology, Sendai Medical Center, National Hospital Organization, Japan, ${ }^{3}$ Division of Neurology, Sendai Medical Center, National Hospital Organization, Japan and ${ }^{4}$ Division of Pathology and Laboratory Medicine, Sendai Medical Center, National Hospital Organization, Japan

Received for publication April 4, 2016; Accepted for publication June 14, 2016

Correspondence to Dr. Ryosuke Tashiro, tashiro0329@gmail.com
} 

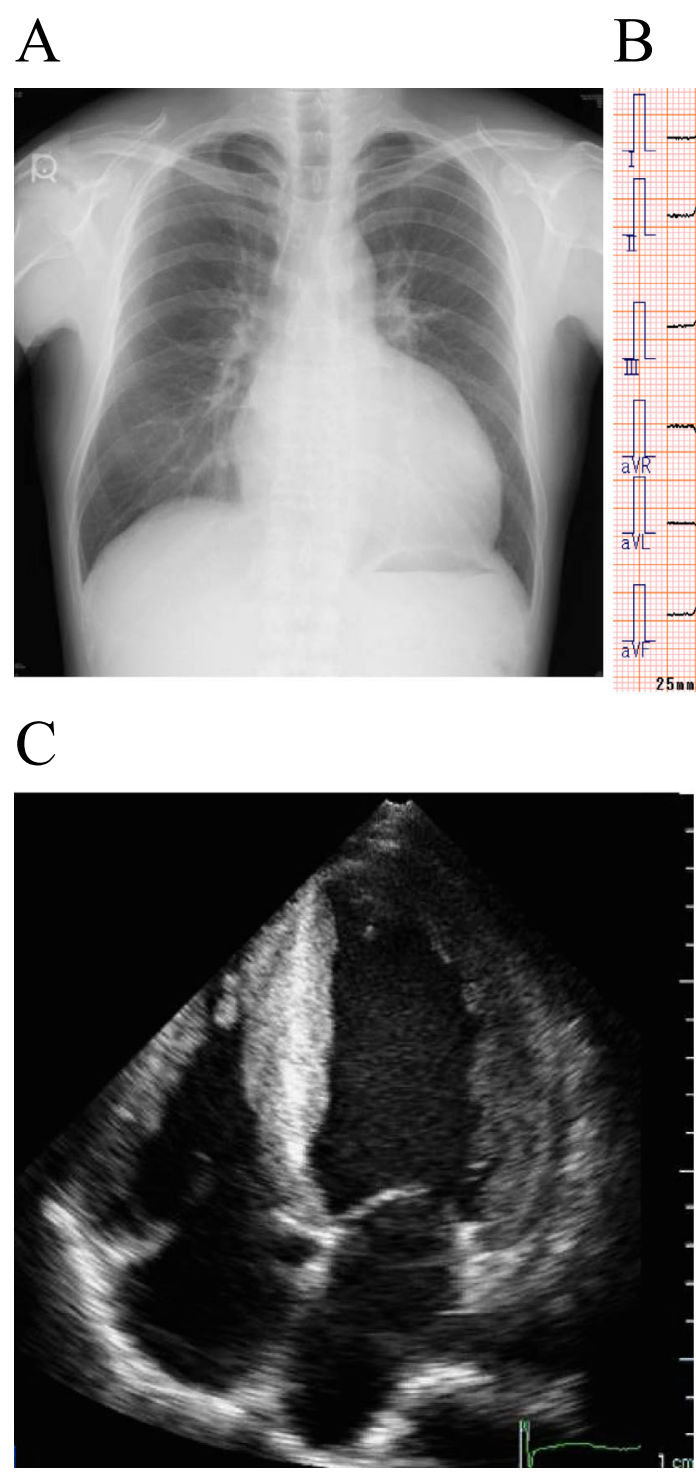

B

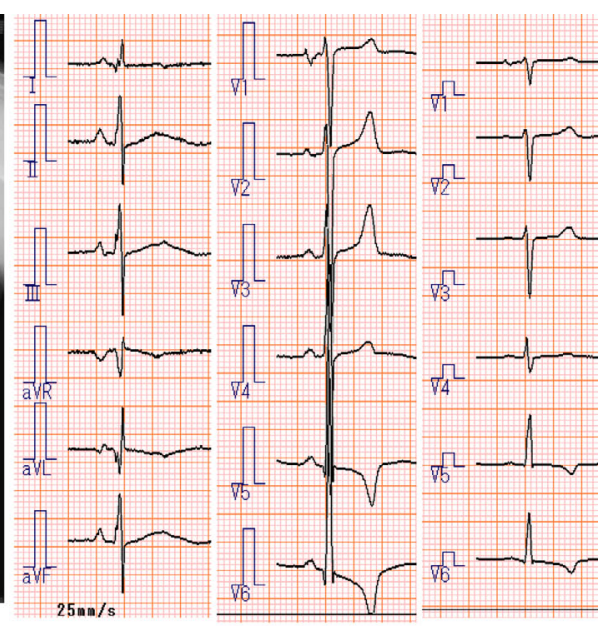

$\mathrm{D}$

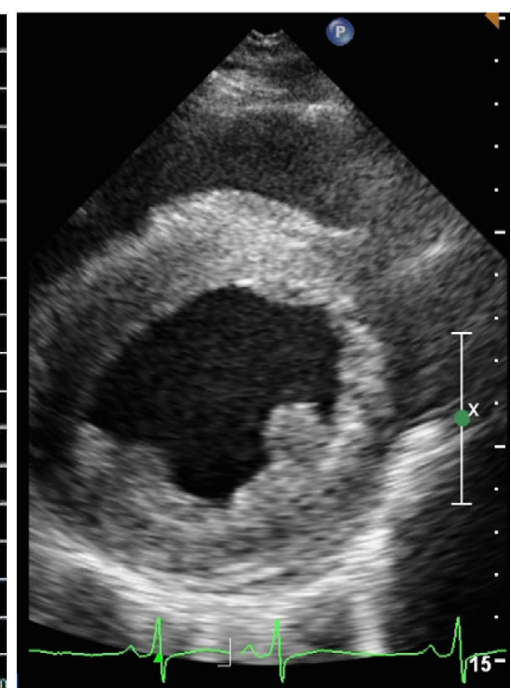

Figure 1. The results of the physiological examinations. (A) Chest radiography reveals cardiomegaly (cardiothoracic ratio, 59\%) and mild pulmonary congestion. (B) An electrocardiogram reveals a prominent $R$ wave progression in V3-6 and a negative $T$ wave in V5-6. $(C$, D) Transthoracic echocardiograms reveal the thickening of the left and right ventricle walls. The left ventricle ejection fraction is $40 \%$.

(4LSB), there was no jugular venous distention, and his breathing sounds were normal. Hepatomegaly and splenomegaly were not present, and there was no edema in his extremities. Mild exophoria and intellectual deterioration were observed.

Chest radiography revealed an increase in the cardiothoracic ratio (59\%) and mild pulmonary congestion (Fig. 1A). Twelve-lead electrocardiography detected a normal sinus rhythm and a high amplitude $\mathrm{R}$ wave with an inverted $\mathrm{T}$ wave in the left lateral precordial leads (Fig. 1B). Transthoracic echocardiography detected thickening of the LV walls (septum, $16 \mathrm{~mm}$; posterior wall, $16 \mathrm{~mm}$ ), as well as thickening of the right ventricle (RV) free wall, the slight dilatation of the LV (LV end-diastolic diameter, $54 \mathrm{~mm}$ ) and diffuse LV systolic dysfunction (ejection fraction, 40\%; using Simpson's method) (Fig. 1C and D). The pressure gradient of the tricuspid regurgitation was $47 \mathrm{mmHg}$.

His levels of troponin $\mathrm{T}$ and $\mathrm{N}$-terminal pro-brain natriuretic peptide were elevated to $0.107 \mathrm{ng} / \mathrm{mL}$ and $1,616 \mathrm{pg}$ / $\mathrm{mL}$, respectively. His fasting glucose level was $172 \mathrm{mg} / \mathrm{dL}$ and his glycated hemoglobin was $7.0 \%$. The resting serum and cerebrospinal fluid lactate concentrations had increased to $27.0 \mathrm{mg} / \mathrm{dL}$ (normal range, $3-17 \mathrm{mg} / \mathrm{dL}$ ) and $31.8 \mathrm{mg} / \mathrm{dL}$ (normal range, 14-21 mg/dL), respectively. Brain computed tomography and magnetic resonance imaging revealed prominent calcification in the bilateral basal ganglia and cerebellar atrophy. Mitochondrial cDNA sequencing detected a c.A3243G mutation, and the patient was diagnosed with mitochondrial disease.

To investigate the cause of his cardiac dysfunction, cardiac catheterization was performed after medical treatment. His pulmonary artery pressure was normal $(31 / 11 \mathrm{mmHg}$, 


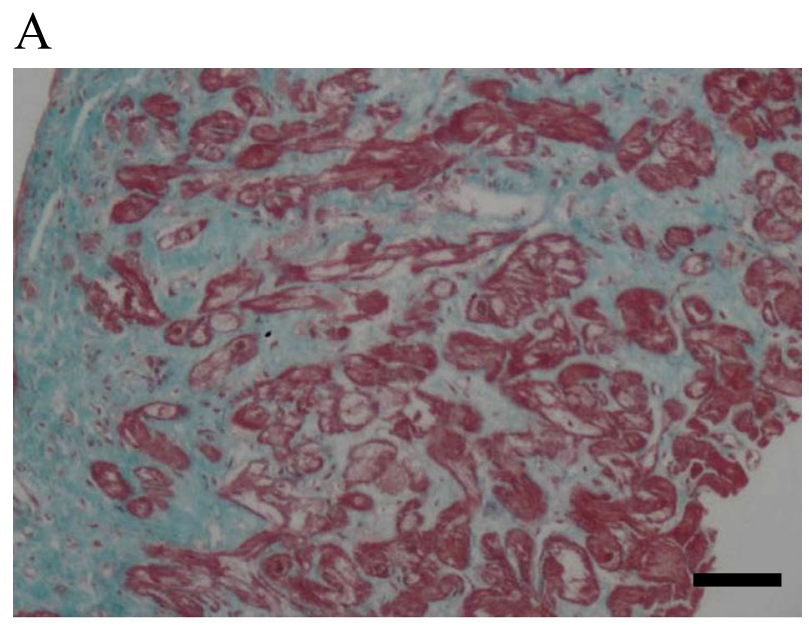

Scale bar: $10 \mu \mathrm{m}$

B

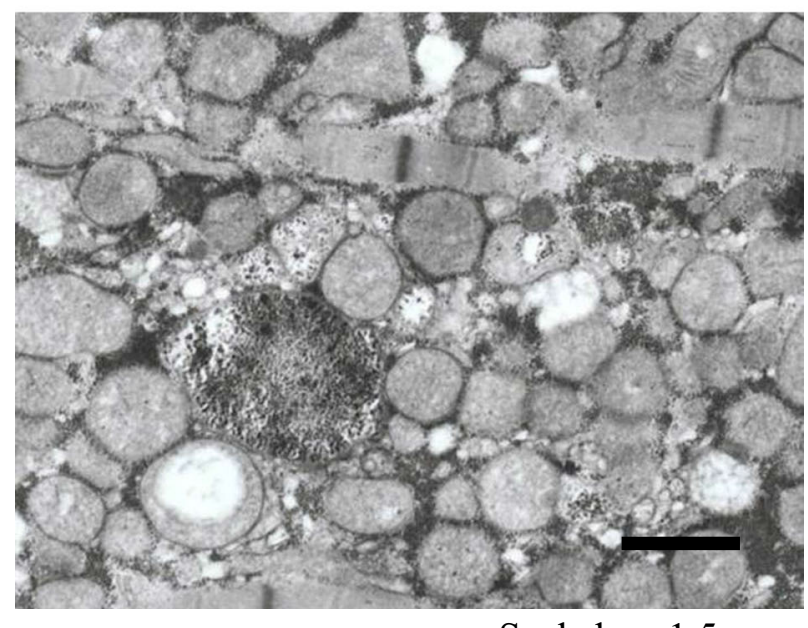

Scale bar: $1.5 \mu \mathrm{m}$

Figure 2. The histopathological findings. (A) A light microscopy image reveals interstitial fibrosis and hypertrophic myocytes with vacuoles (Elastica-Masson staining, 100x). (B) An electron microscopy image reveals an increased number of mitochondria with variable sizes and shapes.

mean $18 \mathrm{mmHg}$ ) with an increase in the LV end-diastolic pressure $(20 \mathrm{mmHg})$. His cardiac index was preserved (3.08 $\mathrm{L} / \mathrm{min} / \mathrm{m}^{2}$ ), and the coronary angiography findings were normal. Left ventriculography revealed diffuse LV hypokinesis (ejection fraction, 27\%). Endocardial biopsy was performed to obtain a sample of the interventricular RV wall. The microscopic findings revealed the rupture and degeneration of the myocardial fibers, which were surrounded by fibrosis (Fig. 2A). Electron microscopy detected an increase in the number of mitochondria, which varied in size and morphology (Fig. 2B).

The patient subsequently underwent resting ${ }^{99 \mathrm{~m}} \mathrm{Tc}-\mathrm{MIBI}$ cardiac scintigraphy (600 MBq), and single-photon emission computed tomography (SPECT) images were obtained at 60 minutes (the early phase) and 240 minutes (the delayed phase) after the injection (4). In the early phase, the uptake of ${ }^{99 m}$ Tc-MIBI did not decrease (Fig. 3A). The heart-to- mediastinum ratio was 2.89 in the early phase and 2.79 in the delayed phase, respectively. The global washout rate in the delayed phase increased by $25 \%$ (normal range: $11 \pm$ $5 \%$ ). The uptake of ${ }^{99 \mathrm{~m}} \mathrm{Tc}-\mathrm{MIBI}$ in the RV free wall appeared to be enhanced in the early phase (Fig. 3A). ${ }^{123}$ I-BMIPP (111 $\mathrm{MBq}$ ) was administered intravenously, and SPECT images were obtained at 35 minutes after the injection (5). The uptake of ${ }^{123}$ I-BMIPP was decreased in the anterior, septal, and inferior walls (Fig. 3B). CMR was performed to estimate late gadolinium enhancement (LGE), which was observed from the midmyocardium to the epicardium of the anterior wall, as well as in the midmyocardium of the septal and inferior walls (Fig. 3C). The LGE margin was obscure and its intensity was inhomogeneous. The ${ }^{99 \mathrm{~m}} \mathrm{Tc}-\mathrm{MIBI} /{ }^{123} \mathrm{I}$-BMIPP mismatch pattern (non-decreased ${ }^{99 m}$ Tc-MIBI uptake/decreased ${ }^{123}$ I-BMIPP uptake) was confirmed via bull's eye mapping (Fig. 3D).

During the patient's hospitalization, we treated his congestive heart failure with furosemide. We prescribed enalapril $(2.5 \mathrm{mg} /$ day $)$ and carvedilol $(1.25 \mathrm{mg} /$ day $)$ to expect preventive effect for the progression of cardiomyopathy $(6,7)$.

\section{Discussion}

Mitochondrial cardiomyopathies elicit a complicated myocardial dysfunction, which is not fully understood. This is the first report to describe the relationship between myocardial perfusion, metabolism, and morphology, and to reveal a unique ${ }^{99 m} \mathrm{Tc}-\mathrm{MIBI} /{ }^{123} \mathrm{I}$-BMIPP mismatch pattern in a patient with mitochondrial cardiomyopathy. Our imaging findings revealed a decrease in the uptake of ${ }^{123}$ I-BMIPP, using LGE in CMR as an anatomical reference. In contrast, previous studies have reported an increase in the uptake of ${ }^{123} \mathrm{I}$ BMIPP in mitochondrial cardiomyopathy (without providing anatomical reference images). The mechanism underlying this observation may be related mitochondrial respiratory chain failure, which leads to the excessive production of nicotinamide adenine dinucleotide (NADH), which then causes an increase in the concentration of glycerol-3phospate (to oxidize the excess NADH) and ultimately leads to increased levels of triglycerides. According to this mechanism, ${ }^{123}$ I-BMIPP (a fatty acid analogue) is incorporated into the cardiomyocytes' pool of triglycerides $(3,8)$. In our patient, the distribution of the areas that showed a low uptake of ${ }^{123}$ I-BMIPP was consistent with the distribution of LGE, which suggests that the low uptake of ${ }^{123}$ I-BMIPP might be correlated with the low number of viable myocytes or fibrosis, rather than an energy production from fatty acid metabolism to the glycolytic pathway (as in other cardiomyopathies). Thus, the extent of the ${ }^{123}$ I-BMIPP uptake may depend on the balance between the mitochondrial respiratory chain failure and the viable myocyte mass, whereby the loss of a certain number of myocytes might outweigh the increased uptake of ${ }^{123}$ I-BMIPP.

The uptake of ${ }^{99 m}$ Tc-MIBI in the early phase was not de- 


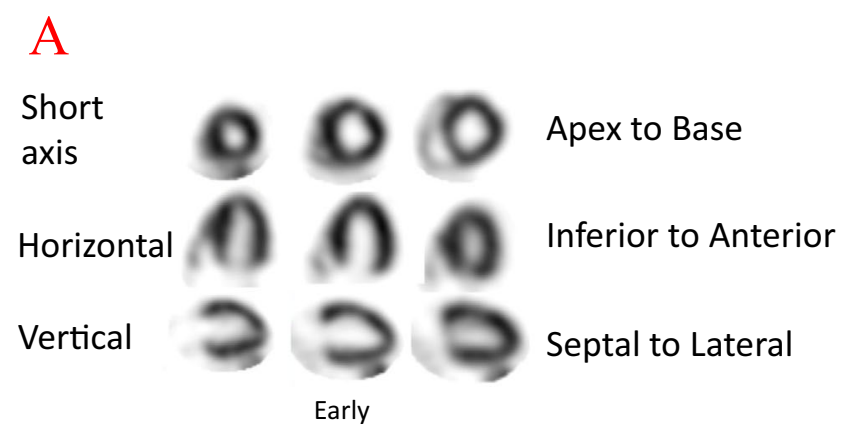

B

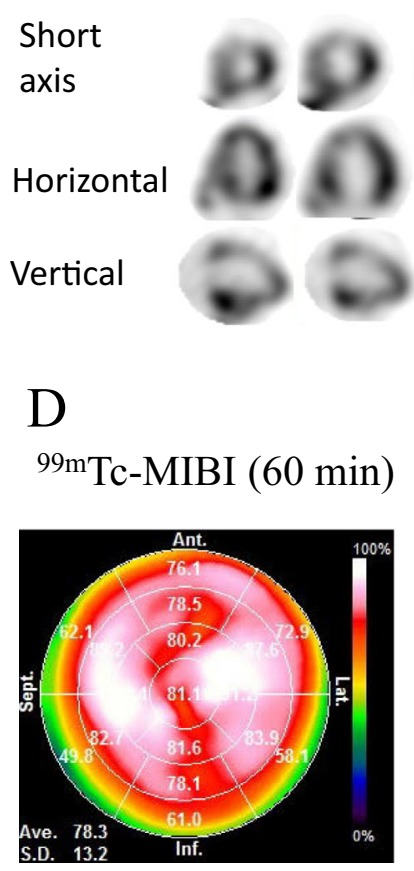

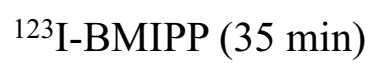

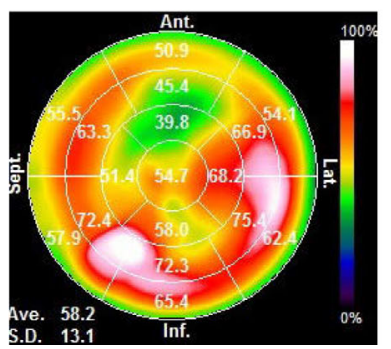

Apex to Base

Inferior to Anterior

Septal to Lateral
C

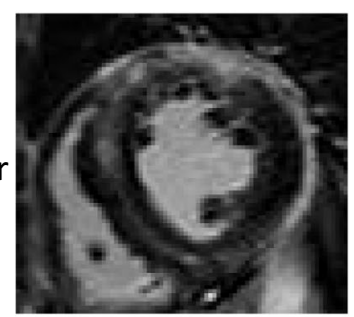

Figure 3. Cardiac scintigraphy and cardiac magnetic resonance imaging. (A) The uptake of ${ }^{99 \mathrm{~m}}$ TcMIBI is not decreased in the early phase. (B) The uptake of ${ }^{123} \mathrm{I}-\mathrm{BMIPP}$ is decreased in the anterior, septal, and inferior walls. (C) Late gadolinium enhancement (LGE) is observed from the midmyocardium to the epicardium of the anterior wall, as well as in the midmyocardium of the septal and inferior walls. (D) Bull's eye mapping shows the ${ }^{99 \mathrm{~m}} \mathrm{Tc}-\mathrm{MIBI} /{ }^{123} \mathrm{I}-\mathrm{BMIPP}$ mismatch pattern (non-decreased ${ }^{99 m}$ Tc-MIBI uptake/decreased ${ }^{123}$ I-BMIPP uptake). ${ }^{99 m}$ Tc-MIBI: technetium-99m methoxyisobutylisonitrile. ${ }^{123}$ I-BMIPP: 123-labeled 15-4-iodophenyl-3-(R, S)-methyl-pentadecanoic acid.

creased in this case. One of the interpretations of the preserved uptake of ${ }^{99 \mathrm{~m}} \mathrm{Tc}-\mathrm{MIBI}$ may be the presence of preserved blood flow and viable myocytes. In this case, the unique LGE pattern (obscure margins and inhomogeneous intensity) may reflect the scattered viable myocytes that were surrounded by fibrosis, which were also detected in our histological examination. This pattern is distinct from the homogeneous LGE pattern that is observed in cases of myocardial infarction. It is possible that the preserved myocytes in the LGE-positive area affect the uptake of ${ }^{99 \mathrm{~m}} \mathrm{Tc}$ MIBI. The volume effect of the thickening LV wall might also explain the non-decreased uptake of ${ }^{99 \mathrm{~m}} \mathrm{Tc}-\mathrm{MIBI}$ (similar to the effect in patients with hypertrophic cardiomyopathy) and the enhanced uptake of ${ }^{99 \mathrm{~m}} \mathrm{Tc}-\mathrm{MIBI}$ in the RV free wall.
Furthermore, the washout rate for ${ }^{99 \mathrm{~m}} \mathrm{Tc}-\mathrm{MIBI}$ increased in this case, which is consistent with the findings of previous studies $(2,3,8)$. In this context, mitochondrial dysfunction impairs the retention of the ${ }^{99 \mathrm{~m}} \mathrm{Tc}-\mathrm{MIBI}$ tracer in myocytes, which leads to an increased washout rate for ${ }^{99 \mathrm{~m}} \mathrm{Tc}-\mathrm{MIBI}$. Nevertheless, our unique ${ }^{99 \mathrm{~m}} \mathrm{Tc}-\mathrm{MIBI} /{ }^{123} \mathrm{I}$-BMIPP mismatch pattern (non-decreased ${ }^{99 \mathrm{~m}} \mathrm{Tc}-\mathrm{MIBI}$ uptake/decreased ${ }^{123} \mathrm{I}-$ BMIPP uptake) is distinct from the patterns in previous reports (decreased ${ }^{99 \mathrm{~m}} \mathrm{Tc}-\mathrm{MIBI}$ uptake/increased ${ }^{123} \mathrm{I}$-BMIPP uptake) $(2,3)$. The ${ }^{99 \mathrm{~m}} \mathrm{Tc}-\mathrm{MIBI} /{ }^{123} \mathrm{I}-\mathrm{BMIPP}$ mismatch pattern in the present case can be considered to be a nonspecific phenomenon in patients with dilated cardiomyopathy (9). This discrepancy may be related to the stages or diverse phenotypes of mitochondrial cardiomyopathy. 
In conclusion, multimodality imaging allows us to evaluate the relationship between myocardial perfusion, metabolism, and morphology. Further studies are needed to elucidate the complex processes of myocardial damage in patients with mitochondrial cardiomyopathy.

The authors state that they have no Conflict of Interest (COI).

\section{References}

1. Meyers DE, Basha HI, Koenig MK. Mitochondrial cardiomyopathy: pathophysiology, diagnosis, and management. Tex Heart Inst J 40: 385-394, 2013.

2. Matsuo S, Nakajima K, Kinuya S, et al. Cardiac scintigraphic findings of mitochondrial myopathy, encephalopathy, lactic acidosis and stroke-like episodes: A case report. Exp Clin Cardiol 13: 93-95, 2008.

3. Ikawa M, Kawai Y, Arakawa K, et al. Evaluation of respiratory chain failure in mitochondrial cardiomyopathy by assessments of ${ }^{99 \mathrm{~m}} \mathrm{Tc}-\mathrm{MIBI}$ washout and ${ }^{123} \mathrm{I}$-BMIPP/ ${ }^{99 \mathrm{~m}} \mathrm{Tc}-\mathrm{MIBI}$ mismatch. Mitochondrion 7: 164-170, 2007.

4. Hayashi D, Ohshima S, Isobe S, et al. Increased ${ }^{99 m} \mathrm{Tc}-S e s t a m i b i$ washout reflects impaired myocardial contractile and relaxation reserve during dobutamine stress due to mitochondrial dysfunction in dilated cardiomyopathy patients. J Am Coll Cardiol 61: 20072017, 2013.

5. Matsuo S, Nakamura Y, Takahashi M, et al. Myocardial metabolic abnormalities in hypertrophic cardiomyopathy assessed by iodine123-labeled beta-methyl-branched fatty acid myocardial scintigraphy and its relation to exercise-induced ischemia. Jpn Circ J 62: 167-172, 1998.

6. Packer M, Bristow MR, Cohn JN, et al. The effect of carvedilol on morbidity and mortality in patients with chronic heart failure. N Engl J Med 334: 1349-1355, 1996.

7. The SOLVD Investigators. Effect of enalapril on survival in patients with reduced left ventricular ejection fractions and congestive heart failure. N Engl J Med 325: 293-302, 1991.

8. Takagi Y, Suzuki K, Kou S, et al. A case of mitochondrial encephalopathy detected dysfunction of cardiac muscle of mitochondria by cardiac nuclear medicine. Shinzo 46: 629-638, 2014 (in Japanese, Abstract in English).

9. Yoshida A, Takano H, Asai K, et al. Comparison pf perfusionmetabolism mismatch in ${ }^{99 m}$ Tc-MIBI and ${ }^{123}$ I-BMIPP scintigraphy with cardiac magnetic resonance in patients with dilated cardiomyopathy. J Card Fail 19: 445-453, 2013.

The Internal Medicine is an Open Access article distributed under the Creative Commons Attribution-NonCommercial-NoDerivatives 4.0 International License. To view the details of this license, please visit (https://creativecommons.org/licenses/ by-nc-nd/4.0/).

(C) 2017 The Japanese Society of Internal Medicine http://www.naika.or.jp/imonline/index.html 